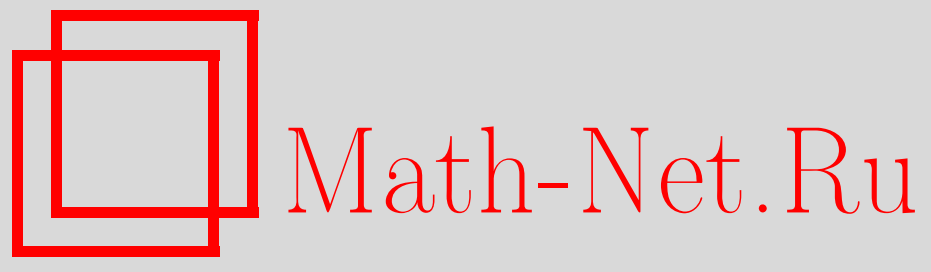

А. А. Давыдов, В. М. Закалюкин, Управляемость нелинейных систем: типичные особенности и их устойчивость, УМН, 2012, том 67, выпуск 2, 65-92

DOI: https://doi.org/10.4213/rm9468

Использование Общероссийского математического портала Math-Net.Ru подразумевает, что вы прочитали и согласны с пользовательским соглашением http://www . mathnet.ru/rus/agreement

Параметры загрузки:

IP : 3.93 .64 .190

26 апреля 2023 г., $16: 26: 44$

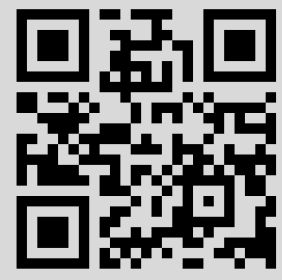




\title{
Управляемость нелинейных систем: типичные особенности и их устойчивость
}

\author{
А. А. Давыдов, В. М. Закалюкин
}

Дан обзор современного состояния приложений теории особенностей к анализу типичных особенностей управляемости нелинейных систем на многообразиях.

Библиография: 40 названий.

Ключевые слова: управляемость, особенность, устойчивость, классификация.

\section{СОДЕРЖАНИЕ}

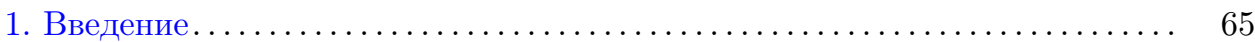

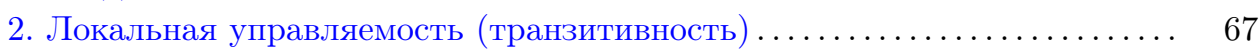

3. Особенности границы зоны локальной транзитивности ............ 70

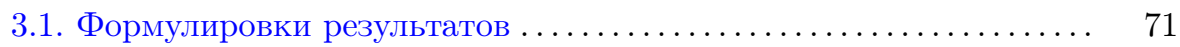

3.2. Преобразования Лежандра и опорные гиперплоскости ........ 78

4. Особенности нелокальной управляемости ................... 82

4.1. Гёльдеровость границы достижимости............... 82

4.2. Особенности нелокальной управляемости в двумерном случае.. 83

4.3. Структурная устойчивость управляемых систем на поверхностях 86

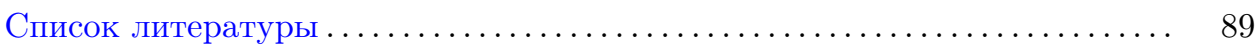

\section{1. Введение}

Активное применение методов и достижений теории особенностей к исследованию проблем теории управления и оптимизации началось в 70-е годы прошлого века, когда идеи этой тогда быстро развивающейся области математики находили новые применения. $\mathrm{K}$ числу первых работ, где была прямо обозначена необходимость развития приложений теории особенностей к задачам управления и оптимизации, несомненно, относится статья С. Смейла [1]. Сразу после этой работы В. И. Арнольд сформулировал для участников своего семинара несколько тем и конкретных задач в этом направлении. Сейчас эти задачи опубликованы в книге [2]. Отметим, в частности, задачи 1972-2

Работа выполнена при поддержке АВЦП РНПВШ (проект 2.1.1/5568). 
и 1973-2 о выпуклых оболочках, задачи 1973-1 и 1973-3 об особенностях в дифференциальных играх и экономических моделях. После этого в течение примерно десяти лет в рамках такого подхода были получены серьезные продвижения в таких важных задачах, как классификация типичных особенностей волновых фронтов [3], [4], выпуклых оболочек замкнутых подмногообразий в линейных пространствах [5]-[9], функций минимума и минимакса семейства функций [10]-[12], как анализ границы достижимости типичных управляемых систем [13], исследование особенностей типичных полисистем [14] и многое другое.

Общность полученных результатов показала эффективность новых подходов, особенно при анализе нелинейных задач. Они стимулировали развитие геометрических методов в математической теории управления - области, в которую естественно могут быть включены все виды геометрий [15], [16].

В этой работе мы обсуждаем современные результаты, связанные только с одним кругом вопросов: с локальной и нелокальной управляемостью нелинейных систем общего положения, с особенностями этой управляемости и устойчивости особенностей к малому возмущению типичной системы.

С геометрической точки зрения управляемая система на гладком многообразии $M$, называемом фазовым пространством, определяется так: в пространстве $T_{x} M$ касательных векторов в каждой точке $x \in M$ задается свое подмножество $I(x)$, называемое индикатрисой или множеством допустимых скоростей.

Допустимым движением системы называется абсолютно непрерывное отображение $t \mapsto x(t)$ промежутка числовой оси (времени) в фазовое пространство системы, в каждой точке дифференцируемости которого скорость движения $\dot{x}(t)$ принадлежит выпуклой оболочке индикатрисы скоростей $I(x(t))$ в этой точке.

Во многих примерах индикатрисы задаются с помощью семейства гладких отображений $V_{x}: U \rightarrow T_{x} M, V_{x}: u \mapsto v(x, u)$, некоторого многообразия $U$, имеющего хотя бы две различные точки и называемого пространством (значений) управления. Отображения зависят от точки $x$ многообразия как от параметра:

$$
I(x)=\{v(x, u), u \in U\} .
$$

В таком случае в локальных координатах $x$ на многообразии управляемую систему записывают в стандартном виде (см. [16])

$$
\dot{x}=v(x, u) \text {. }
$$

Следуя общим принципам теории особенностей, мы рассматриваем пространство управляемых систем, отождествляя его с пространством отображений $v$ и снабжая его гладкой или достаточно гладкой тонкой топологией Уитни [17]. Типичная система (или система общего положения) - это система из открытого всюду плотного подмножества в этом пространстве в выбранной топологии. Свойства, присущие системам общего положения, и составляют предмет нашего исследования.

Как только задана управляемая система, возникает вопрос о ее управляемости, локальной и нелокальной. А именно, в какие конечные точки у можно 
попасть с помощью допустимого движения из заданного начального состояния $x$, или наоборот, из каких точек можно прийти в $x$. Этот вопрос имеет, конечно, многочисленные варианты и уточнения.

В качестве примера красивого классического результата такого типа упомянем теорему Лассаля: линейная система

$$
\dot{x}=A x+B u, \quad x \in \mathbb{R}^{n},
$$

с постоянными матрицами $A, B$ и множеством управлений, содержащим в $\mathbb{R}^{m}$ окрестность нуля, управляема в нуле при условии, что ранг матрицы со столбцами $B, A B, \ldots, A^{n-1} B$ равен $n$. Другими словами, найдется допустимое движение из нуля в любую точку и из любой точки в нуль. Локальную управляемость в нуле для общей дифференцируемой системы $\dot{x}=v(x, u), v(0,0)=0$, линеаризация которой в нуле удовлетворяет условию Лассаля, доказал Р. Калман (см. [18]).

Если из $x$ в $y$ имеется достаточное количество допустимых движений, встает вопрос о выборе оптимального движения. Конечно, должен быть задан функционал цены (или качества) на пространстве движений, который и подлежит оптимизации [15], [16]. Приложения теории особенностей к различным задачам оптимального управления и, в частности, применения принципа максимума Понтрягина как необходимого условия оптимальности [16] - большая и интересная тема, к сожалению выходящая за рамки настоящей статьи.

Итак, наша цель - обзор результатов об особенностях локальной и нелокальной управляемости систем. Сначала, в разделе 2, мы вводим понятия локальной транзитивности системы и приводим иллюстрирующие примеры, а затем, в разделе 3, для типичных систем на двух- и трехмерных многообразиях классифицируем особенности замыкания множества точек, где система локально транзитивна. Наконец, в разделе 4 мы обсуждаем особенности нелокальной управляемости типичных систем на гладких замкнутых ориентируемых поверхностях.

Эта работа посвящается светлой памяти Е. Ф. Мищенко, одного из создателей современной теории управления, всегда бывшего в курсе новых результатов и методов исследования. Евгений Фролович имел тесный контакт с В. И. Арнольдом, основателем отечественной школы теории особенностей, приложения достижений которой к анализу управляемости систем и легли в основу этой статьи. Беседы с Евгением Фроловичем, помимо большого счастья от общения с этим выдающимся человеком, всегда были для нас стимулом к работе в этом направлении.

\section{2. Локальная управляемость (транзитивность)}

Управляемая система называется локалъно транзитивной в точке $x$ фазового пространства, если для любой окрестности $W$ этой точки существуют время $T_{W}>0$ и, возможно, меньшая окрестность $\widetilde{W}$ такие, что любые два состояния из $\widetilde{W}$ переводятся одно в другое допустимым движением за время не большее $T_{W}$ без выхода из окрестности $W$. 
Если для любой окрестности $W$ и любого $T>0$ существует такая (меньшая) окрестность $\widetilde{W}$, что любые два состояния из $\widetilde{W}$ переводятся одно в другое допустимым движением за время не большее $T$ без выхода из окрестности $W$, то система называется локально транзитивной за малое время в этой точке.

Множество точек локальной транзитивности системы образует зону или множество локальной транзитивности (соответственно зону локальной транзитивности за малое время).

Понятия локальной транзитивности и локальной транзитивности за малое время различны. Например, бидинамическая система, заданная на плоскости $\mathbb{R}_{x, y}^{2}$ ровно двумя полями скоростей $(1,0)$ и $(x-y, x+y)$, локально транзитивна в нуле, но не за малое время. Зона локальной транзитивности здесь это луч $\{x+y=0, y \geqslant 0\}$, а локальной транзитивности за малое время - этот же луч, но без нуля. При малом возмущении системы эти две зоны незначительно изменяются, но по-прежнему совпадают, за исключением особой точки второго поля. Таким образом, различие этих зон - устойчивая особенность (неустранимая малым возмущением системы).

Еще одну иллюстрацию этих понятий дает двумерная модель А. Д. Мышкиса пловца в потоке воды на плоскости $\mathbb{R}_{x, y}^{2}$, который задается векторным полем $(-x,-\beta y), 0<\beta \in \mathbb{R}$, и имеет единственный сток в нуле для всей плоскости [19], [20]. "Безинерционный” пловец в стоячей воде может плыть в любом направлении со скоростью не больше 1, а в данном потоке воды его индикатрисы скоростей задаются дифференциальным неравенством

$$
(\dot{x}+x)^{2}+(\dot{y}+\beta y)^{2} \leqslant 1
$$

и всюду строго выпуклы.

Это поле индикатрис можно задать управляемой системой, например, если за многообразие управлений принять единичную сферу $u_{1}^{2}+u_{2}^{2}+u_{3}^{2}=1$ в пространстве $\mathbb{R}_{u_{1}, u_{2}, u_{3}}^{3}$, а отображение $v$ в определении индикатрис взять в виде

$$
(x, y) \times\left(u_{1}, u_{2}, u_{3}\right) \mapsto(x, y) \times\left(-x+u_{1},-\beta y+u_{2}\right) .
$$

В области $x^{2}+(\beta y)^{2}<1$ пловец может плыть с ненулевой скоростью в любом направлении, поэтому в каждой из точек этой области система локально транзитивна за малое время (см. [21], [22]). Напротив, в дополнении к замыканию этой области у пловца недостаточно сил, чтобы противостоять потоку, в каждой из точек этой области индикатриса скоростей и нулевая скорость лежат по разные стороны от некоторой прямой, причем индикатриса принадлежит сектору, ограниченному двумя предельными направлениями скоростей. Легко видеть, что в каждой из точек этой области система не является локально транзитивной.

Осталось разобраться с локальной управляемостью системы в точках эллипса $x^{2}+(\beta y)^{2}=1$ - границы зоны локальной транзитивности системы. Оказывается, что в двумерном случае локальная управляемость типичной системы в точках этой границы полностью определяется особенностями поля предельных направлений в этих точках или особенностями сети его интегральных 
кривых - предельных линий, движение системы по которым использует допустимые скорости предельного направления.

Это поле направлений (или сеть кривых) задается дифференциальным уравнением

$$
(x d y-\beta y d x)^{2}\left(x^{2}+(\beta y)^{2}-1\right)=(x d x+\beta y d y)^{2},
$$

не разрешенным относительно производной. Это уравнение не имеет вещественных решений внутри зоны локальной транзитивности, а в каждой внутренней точке дополнения к ней задает два вещественных направления, которые стремятся одно к другому при подходе к границе этой зоны. На границе зоны это приводит к гладкому полю направлений, которое задается уравнением $x d x+\beta y d y=0$ и касается этой границы - эллипса - в его вершинах с первым порядком касания.

При $\beta>2$ уравнение (1) имеет особые точки "сложенные седла" и "узлы" в вершинах $( \pm 1,0)$ и $(0, \pm 1 / \beta)$ соответственно. В этих точках система не является локально транзитивной, ибо вблизи них легко строятся барьеры, которые допустимые движения пловца могут пересекать лишь в одном направлении (см. рис. 1). В каждой из остальных точек границы зоны локальной транзитивности система локально транзитивна за малое время, что нетрудно проверить.

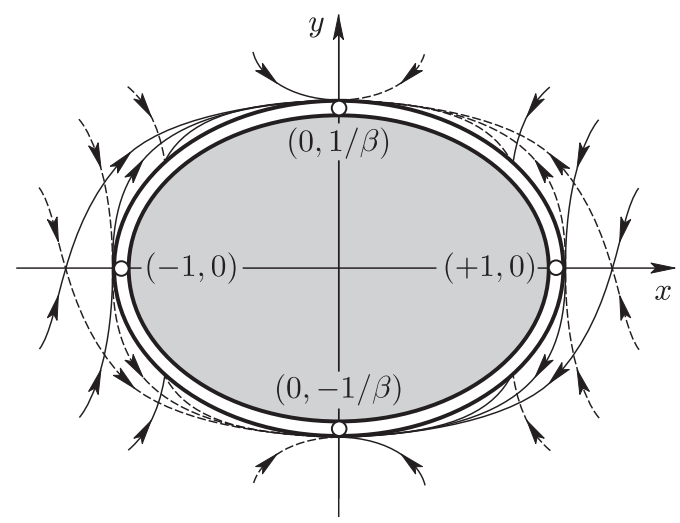

Рис. 1. Локальная транзитивность пловца на плоскости

ЗАмЕчАниЕ 1. Заметим, что для типичных динамических неравенств вида

$$
\left(\dot{x}-v_{1}(x, y)\right)^{2}+\left(\dot{y}-v_{2}(x, y)\right)^{2} \leqslant 1
$$

на плоскости с гладким полем сноса $\left(v_{1}(x, y), v_{2}(x, y)\right)$ и простым движением самого объекта на границе зоны локальной транзитивности может наблюдаться еще только один случай - особая точка "сложенный фокус" поля предельных направлений допустимых скоростей, где система локально транзитивна, но не за малое время [23], [24].

Очевидно, что множество точек, где выпуклая оболочка индикатрисы содержит нулевую скорость и, соответственно, область точек, где эта скорость 
лежит вне выпуклой оболочки, мало изменяются при достаточно малом возмущении управляемой системы. Однако, начиная с размерности 4 фазового пространства, поведение самой границы этих областей при малых возмущениях не изучено. Например, не известно даже, является ли эта граница топологическим подмногообразием, разделяющим эти две области в фазовом пространстве и если да, то устойчиво ли оно к малому возмущению типичной управляемой системы.

Перейдем к описанию конкретных особенностей зон локальной транзитивности для типичных систем в трехмерном пространстве и на плоскости.

\section{3. Особенности границы зоны локальной транзитивности}

Здесь, следуя в основном работам [25]-[28], мы описываем типичные особенности границы множества локальной транзитивности управляемых систем на плоскости и в трехмерном пространстве. Рассмотрены основные виды управляемых систем, а именно, рассмотрены случаи, когда индикатрисы $I(x) \subset T_{x} M$ являются:

- либо гладкими кривыми или поверхностями, вложенными (или погруженными) в линейные пространства $T_{x} M$ касательных векторов в точках $\in M$;

- либо гладкими кривыми или поверхностями с границами и углами, вложенными в пространства $T_{x} M$;

- либо образами гладких отображений общего положения гладкого замкнутого многообразия $U$ размерности не меньшей двух;

- либо, наконец, образами гладких отображений многообразия $U$ с границей и углами.

Напомним, что угол на многообразии - это его подмножество, диффеоморфное координатному углу арифметического пространства. Наличие границ или углов у многообразия $U$ управлений моделирует наличие ограничений (на управления) в виде неравенств. Это весьма распространенный в приложениях вид управляемых систем.

Рассматривая точку $x$ многообразия $M^{n}$ в качестве параметра и зафиксировав некоторую тривиализацию касательного расслоения к $M$, получим семейство подмногообразий $I(x)$, вложенных в $\mathbb{R}^{n}$. Соответственно, границей $\Sigma$ множества локальной управляемости будем считать множество параметров $x$, при которых $0 \in \mathbb{R}^{n}$ принадлежит границе выпуклой оболочки соответствующего подмногообразия $I(x)$. Напомним, что выпуклая оболочка (замкнутого) подмножества аффинного пространства - это пересечение всех замкнутых полупространств, содержащих это подмножество.

Заметим также, что границы выпуклых оболочек гладких подмногообразий общего положения могут иметь особенности. Их классификацию, тесно связанную с особенностями границ зоны локальной транзитивности, мы также обсуждаем ниже. 
3.1. Формулировки результатов. Начнем с простейшего случая управляемой системы на двумерном многообразии $M$, в касательной плоскости каждой точки которого задано множество допустимых скоростей $I(x)$, являющееся гладкой замкнутой кривой.

Напомним [5], что граница выпуклой оболочки типичной гладкой кривой на плоскости в каждой точке является либо ростком гладкой кривой (это росток либо выпуклой кривой, либо прямой); либо ростком кривой, который диффеоморфизмом плоскости можно перевести в росток в нуле графика функции $y=f(x)$, где

$$
f(x)= \begin{cases}0 & \text { при } x \leqslant 0 \\ x^{2} & \text { при } x>0\end{cases}
$$

Если кривая имеет концевые точки, то к указанному списку добавляется особенность, являющаяся графиком функции $g(x)=|x|$.

Для типичного семейства гладких кривых, зависящего от двух параметров, граница $\Sigma$ множества локальной транзитивности может иметь [25] еще только одну новую особенность, по сравнению с особенностями выпуклой оболочки индивидуальной кривой общего положения, а именно указанную выше особенность в нуле графика функции $y=|x|$.

Оказывается, что в точности таким же является и список типичных локальных особенностей (с точностью до диффеоморфизмов плоскости) границы зоны транзитивности для управляемых систем на плоскости, индикатрисы которых являются гладкими кривыми с концевыми точками.

Более того, точно такие же особенности выпуклых оболочек и границ зон локальной транзитивности имеются и в случае, когда индикатрисы являются образами гладких отображений многообразия $U$ произвольной размерности, образующих семейства общего положения. При этом можно считать, что многообразие $U$ имеет границы и углы.

Методы доказательства этих и других фактов обсуждаются ниже.

Перейдем к основному случаю трехмерного многообразия $M$.

Сначала предположим, что индикатриса является $C^{\infty}$-гладкой замкнутой пространственной кривой. Список типичных локальных особенностей выпуклой оболочки кривой в $\mathbb{R}^{3}$ с точностью до диффеоморфизма объемлющего пространства был получен в работах [29] и [6]. Он состоит из первых шести из нормальных форм, перечисленных в приведенной ниже теореме 1.

Чтобы описать особенности границ выпуклых оболочек пространственных кривых с концевыми точками, введем следующие определения.

Замкнутую выпуклую простую кусочно гладкую кривую $\gamma$, лежащую в плоскости $z=1$ пространства $\mathbb{R}^{3}=\{(x, y, z)\}$, назовем простой образующей, если она состоит из перемежающихся отрезков прямых и строго выпуклых дуг и в их общих точках имеет класс $C^{1}$. Кривую $\gamma$ назовем образующей $c k$ углами, если она содержит стороны $k$ углов меньших $\pi$, соединенные между собой выпуклыми частями, состоящими из отрезков и дуг, имеющих класс $C^{1}$ во всех общих концах гладких частей, за исключением вершин углов. Некоторые стороны могут быть общими для двух углов. Заменив в этом определении одну 
из прямолинейных сторон угла на дугу выпуклой кривой, получим определение образующей с $k$ углами и одной особой стороной.

Коническую поверхность, образованную отрезками, один конец которых находится в начале координат, а другой пробегает либо простую образующую $(k=0)$, либо образующую с $k=1,2, \ldots$ углами, одна из сторон которых может быть особой, назовем $k$-конусом.

Заметим, что ростки в начале координат таких конусов имеют функциональные инварианты относительно действия диффеоморфизмов, поскольку касательные векторы в вершине образуют касательный конус, заданный выпуклой кривой на сфере направлений, который подвергается лишь линейным преобразованиям при действии диффеоморфизмов.

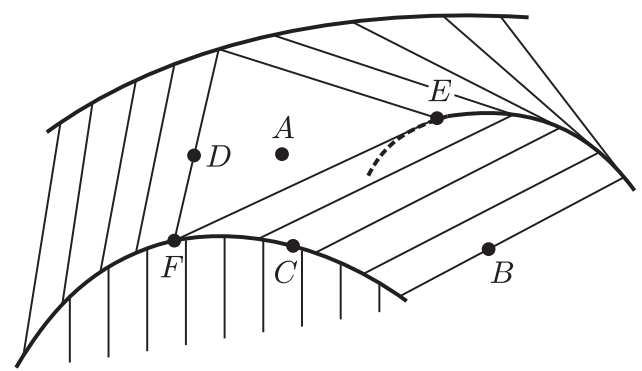

Рис. 2. Особенности "ребро", "сопряжение", "нос" и "корма"

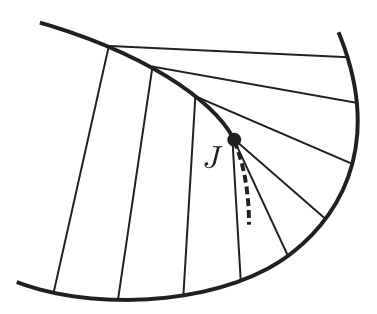

Рис. 3. Особенность "усеченный ласточкин хвост"

Tеорема 1 [27]. Росток в любой точке границы выпуклой оболочки связной пространственной кривой общего положения с конщевыми точками с помощвю диффеоморфизма $\mathbb{R}^{3}$ приводится $к$ ростку в нуле графика одной из следующих функиий $z=f_{i}(x, y)$, где $i=1, \ldots, 7$ (рис. 2-5):

1) $f_{1}=0$ (росток гладкой поверхности);

2) $f_{2}(x, y)=|x|($ ребро $)$;

3) $f_{3}(x, y)=\left\{\begin{array}{ll}0 & \text { при } x \leqslant 0, \\ x^{2} & \text { при } x>0\end{array} \quad\right.$ (сопряжение);

4) $f_{4}(x, y)=\left\{\begin{array}{ll}x^{2} & \text { при } y \leqslant x, x \geqslant 0, \\ y^{2} & \text { при } y \geqslant 0, y \geqslant x, \\ 0 & \text { при } y \leqslant 0, x \leqslant 0\end{array} \quad\right.$ (нос);

5) $f_{5}(x, y)=\left\{\begin{array}{ll}0 & \text { nрu } y \leqslant 0, x \leqslant 0, \\ x^{2} & \text { nрu } y \leqslant-x, x \geqslant 0, \\ y^{2} & \text { nрu } y \geqslant 0, y \leqslant-x, \\ \frac{1}{2}\left(x^{2}+y^{2}\right)-y-x & \text { nрu } x+y \geqslant 0\end{array} \quad\right.$ (корма);

6) $f_{6}(x, y)=\min _{z \in \mathbb{R}}\left\{z^{4}+x z^{2}+y z\right\} \quad$ (усеченный ласточкин хвост); 
7) $f_{7}(x, y)=\left\{\begin{array}{ll}y^{2}+x & \text { nри } x \geqslant 0, \\ y^{2} & \text { nри } y \leqslant 0, x \leqslant 0, \\ (1-x) y^{2} & \text { nри } y \geqslant 0, x \leqslant 0\end{array} \quad\right.$ (изгиб);

либо к ростку в нуле $k$-конуса при $k=0,1,2$.

ЗАмечАниЕ 2. Типичные особенности выпуклой оболочки замкнутой пространственной кривой (без концевых точек) составляют список первых 6 классов из этой теоремы.

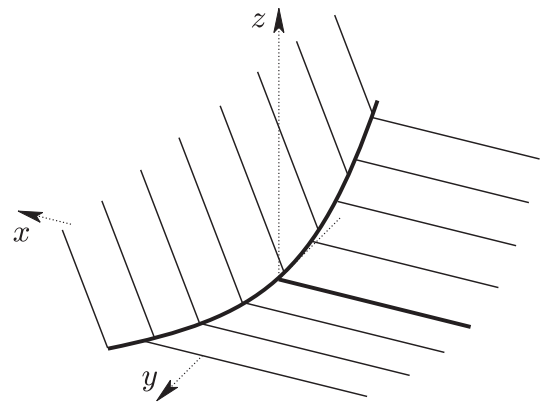

Рис. 4. Особенность "изгиб"

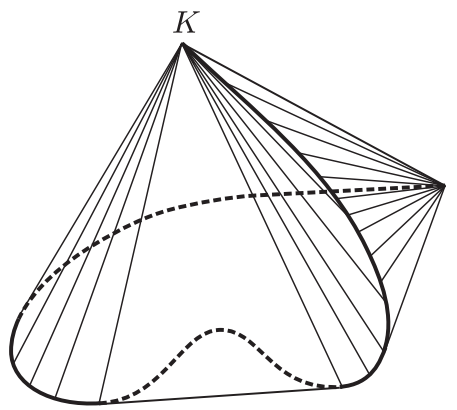

Рис. 5. Особенность " $k$-конус"

ЗАмЕчАниЕ 3. Гладкая поверхность 1) может быть либо строго выпуклой, либо развертывающейся (рис. 2 , точка $B$ ), либо плоской (рис. 2, точка $A$ ); ребро 2) возникает в типичной точке самой исходной кривой (рис. 2 , точка $C$ ); особенность 3) появляется, в частности, в точках сопряжения развертывающейся поверхности и части плоскости (рис. 2 , точка $D$ ); ростки 4 ), 5 ) отвечают вершинам $E$ и $F$ плоских треугольников, возникающих неустранимым образом на границе выпуклой оболочки. Вершина конуса совпадает с концевой точкой (рис. 5 , точка $K$ ). Усеченный ласточкин хвост возникает в точке, касательная в которой пересекает кривую еще в одной точке (рис. 3 , точка $J$ ).

Типичные локальные особенности границы множества транзитивности управляемой системы на трехмерном многообразии с индикатрисой, являющейся замкнутой пространственной кривой, следующие.

Теорема 2. Для типичного семейства гладких кривых $r_{m}: S^{1} \rightarrow \mathbb{R}^{3}$, зависящего от трехмерного параметра $m=(x, y, z) \in \mathbb{R}^{3}$, все локальные особенности границы $\Sigma$ множества транзитивности, с точностью до диффеоморфизма $\mathbb{R}^{3}$, образуют список, состоящий из ростков 1)-7) из теоремъ 1 , а также ростков 9), 12), 14) из следующей ниже теоремы 3.

Для пространственной кривой с концевыми точками типичные особенности множества транзитивности следующие.

Теорема 3. Для семейства общего положения связных пространственных кривых с кониевыми точками, зависящего от трех параметров, росток 
границы $\Sigma$ множества транзитивности во всякой ее точке дифбеоморфизмом приводится либо к одному из ростков 1)-7) грании, выпуклых оболочек типичных кривых в $\mathbb{R}^{3}$, перечисленных в теореме 1 , либо $к$ ростку в нуле графика одной из следующих функиии:

8) $f_{8}(x, y)= \begin{cases}0 & \text { при } x \leqslant 0, y \leqslant 0, \\ y^{2} & \left.\text { при } y^{2} \geqslant x, y \geqslant 0, \quad \text { (срез }\right) \text {; } \\ x & \text { при } y \leqslant \sqrt{x}, x \geqslant 0\end{cases}$

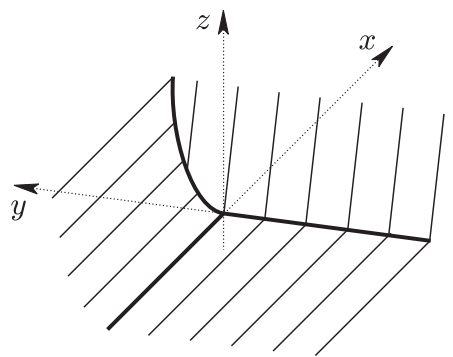

Рис. 6. Особенность "срез"

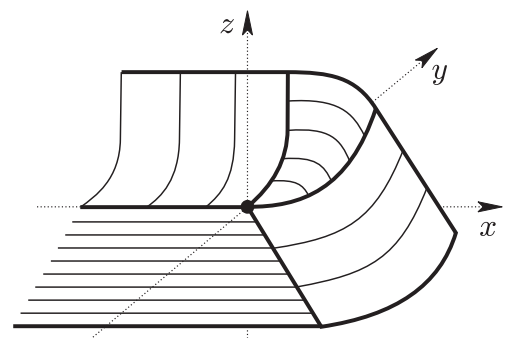

Рис. 7. Особенность "ваза"

9) $f_{9}(x, y)= \begin{cases}0 & \text { nрu } y \leqslant 0, x+\alpha y \leqslant 0, \\ \frac{(x+\alpha y)^{2}}{1+\alpha^{2}} & \text { nрu } y \leqslant \alpha x, x+\alpha y \geqslant 0, \alpha \neq 0, \\ y^{2} & \text { nрu } y \geqslant 0, x \leqslant 0, \\ x^{2}+y^{2} & \text { nрu } x \geqslant 0, y \geqslant \alpha x\end{cases}$

(сопряжение четырех поверхностей - ваза);

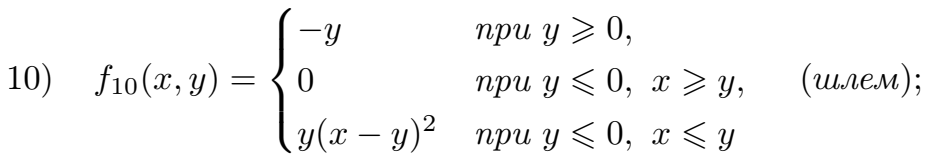

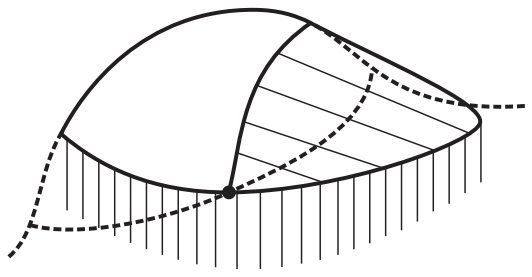

Рис. 8. Особенность "шлем"

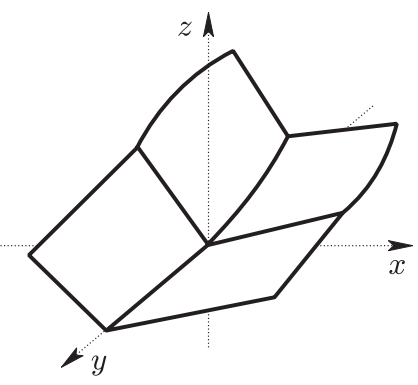

Рис. 9. Особенность "бабочка"

11) $f_{11}(x, y)=\left\{\begin{array}{ll}|x| & \text { при } y \geqslant 0, \\ |x|+y^{2} & \text { при } y<0\end{array} \quad\right.$ (бабочка); 
либо к ростку в нуле обгединения трех поверхностей с краем, заданных условиями

12)

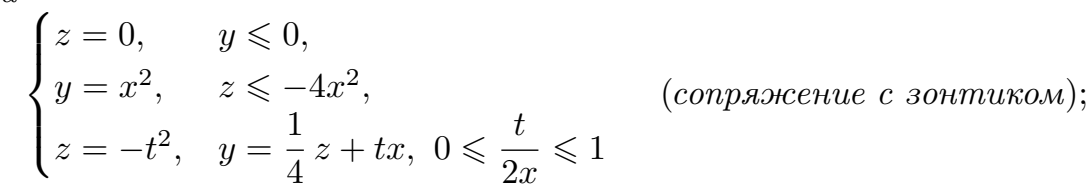

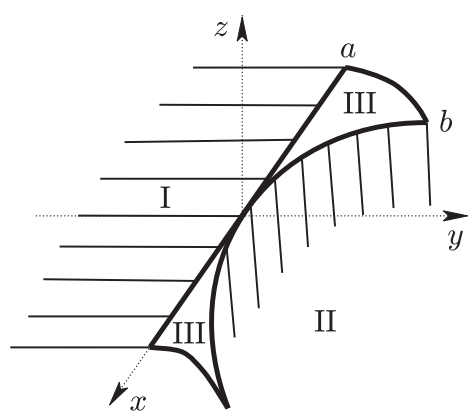

Рис. 10. Особенность "сопряжение с зонтиком"

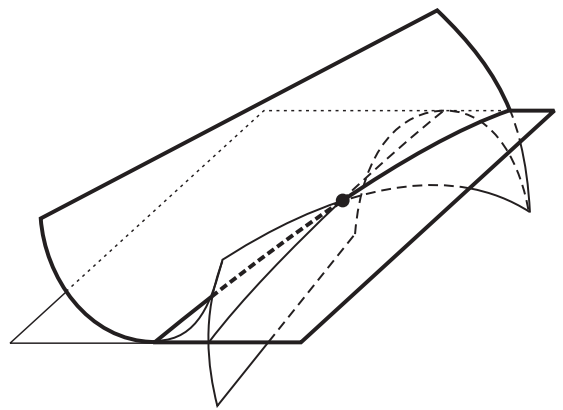

Рис. 11. Особенность "тетрадь"

либо к ростку в нуле двух поверхностей с общим краем, заданных условиями

$$
\begin{cases}z=0 & \text { при } y, x \geqslant 0 \text { или при } x \leqslant 0, y \geqslant \frac{1}{4} x^{2}, \\ z=2 t^{3}+x t^{2} & \text { при } 3 t^{2}+2 t x+y=0, t \geqslant \max \left\{0,-\frac{x}{2}\right\},\end{cases}
$$

либо к ростку боковой поверхности п-угольной пирамиды в ее вершине при $n=3,4,5$, m.е. $к$ ростку границы области, заданной неравенствами

$$
\begin{aligned}
x, y, z & \geqslant 0 & \text { nрu } n & =3, \\
14) & x, y, z \geqslant 0, \quad z-x+y \geqslant 0 & \text { nрu } n & =4, \\
x, y, z & \geqslant 0, \quad z-x+y \geqslant 0, \quad z-\alpha x-\beta y \geqslant 0 & \text { nрu } n & =5
\end{aligned}
$$

(при $n=5$ нормальная форма имеет два числовых инварианта $\alpha$ и $\beta$ );
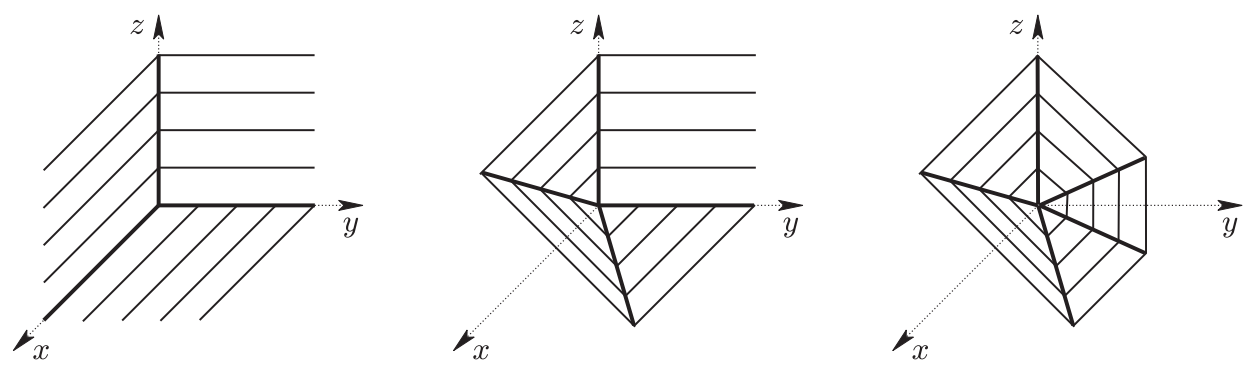

Рис. 12. Особенности "пирамиды"

либо, наконеи, $к$ ростку $k$-конуса с $k=0,1,2$. 
ЗАМЕчАНИЕ 4 . Края $a, b$ поверхностей I и II ростка 12) заданы уравнениями $z=0, y=0$ и $y=x^{2}, z=-4 x^{2}$. Эти кривые касаются друг друга в единственной общей точке - начале координат. Поверхность III представляет собой часть зонтика Уитни, ограниченного этими же краями и касающегося вдоль них поверхностей I и II (рис. 9).

Граница $H\left(I_{x}\right)$ выпуклой оболочки типичной $C^{\infty}$-гладкой поверхности $I_{x}$, может иметь особенности, принадлежащие только классу гладкости $C^{1}$. Типичные локальные особенности $H\left(I_{x}\right)$ [5] - это либо ростки гладкой поверхности, либо росток $C^{1}$-гладкой поверхности "сопряжение", либо "ваза", либо "усеченный ласточкин хвост".

Для поверхностей с границей и углами верны следующие утверждения.

TеОРема 4. Список типичных локальных особенностей границы выпуклой оболочки гладкой компактной поверхности с гладкой границей в $\mathbb{R}^{3}$, с точностъю до диффеоморфизма, состоит из особенностей 1)-7), 9), 11).

ТЕОРема 5. Типичные локалъные особенности выпуклых оболочек гладких компактных поверхностей с границей и углами в $\mathbb{R}^{3}$ - это либо особенности 1)-7), 9), 10), либо $k$-конусы с любъм $k$.

Теорема 6. Для семейства общего положения гладких компактных поверхностей в $\mathbb{R}^{3}$, зависящего от трехмерного параметра, локальные особенности граничь множества локальной транзитивности, с точностъю до дифбеоморфизма пространства параметра, исчерпываются списком ростков 1)-6), 9), 12), 14) нормальных форм.

Теорема 7. Для семейства общего положения гладких компактных поверхностей с гладким краем, зависящего от трехмерного параметра, список локальных особенностей границы множества локальной транзитивности, с точностью до дифбеоморфизма пространства параметра, состоит из особенностей 1)-14).

ТЕОРема 8. Для семейства общего положения гладких компактных поверхностей с краем и углами, зависящего от трехмерного параметра, список локальных особенностей границы зоны локальной транзитивности, с точностью до диффеоморфизма пространства параметра, состоит из всех особенностей: 1)-14) и $k$-конусов с произвольным $k=1,2, \ldots$.

Рассмотрим, наконец, случай, когда индикатрисы в трехмерном пространстве являются образами гладких отображений заданного компактного $n$-мерного многообразия $U^{n}$ управлений.

Теорема 9. Список локальных особенностей границы выпуклой оболочки образа типичного гладкого отображения $V: U^{n} \rightarrow \mathbb{R}^{3}$ гладкого $n$-мерного компактного многообразия $U^{n}$, с точностью до дифбеоморфизма пространства образа, совпадает со списком локалъных особенностей границы выпуклой оболочки компактной пространственной кривой при $n=1$ или соответственно со списком локальных особенностей границы выпуклой оболочки гладкой компактной поверхности при $n>2$. При $n=2 \kappa$ списку локальных особенностей 
границы выпуклой оболочки гладкой замкнутой поверхности добавляется особенность 12) "сопряжение с зонтиком" из теоремы 3.

ТЕОРема 10. Для семейства общего положения гладких отображений $V$ : $U^{n} \rightarrow \mathbb{R}^{3}$ гладкого компактного многообразия $U^{n}$ размерности $n \geqslant 3$, зависящего от трехмерного параметра, список локальных особенностей границы зоны локальной транзитивности, с точностью до диффеоморфизма пространства параметра, совпадает со списком ростков 1)-6), 9), 12), 14) нормальных форм из теоремы 6.

ТЕОРема 11. Список локальных особенностей границы выпуклой оболочки образа типичного отображения $V: U^{n} \rightarrow \mathbb{R}^{3}$ гладкого $n$-мерного компактного многообразия $U^{n}$ с границей и углами, с точностью до дифбеоморфизма пространства-образа, совпадает со списком локальных особенностей границы выпуклой оболочки компактной пространственной кривой с концевыми точками при $n=1$ или соответственно со списком локальных особенностей границы выпуклой оболочки гладкой компактной поверхности с границей и углами при $n>2$. При $n=2 \kappa$ списку добавляется особенность 12) "сопряжение с зонтиком" из теоремы 3 .

ТеОрема 12. Для семейства общего положения гладких отображений $V$ : $U^{n} \rightarrow \mathbb{R}^{3}$ гладкого $n$-мерного компактного многообразия $U^{n}$ с границей и углами, зависящего от трехмерного параметра, список локальных особенностей границы зоны локальной транзитивности, с точностью до диффеоморфизма пространства параметра, совпадает со списком типичных локальных особенностей границы выпуклой оболочки гладкой компактной пространственной кривой с концевыми точками при $n=1$ или соответственно со списком типичных локальных особенностей границь выпуклой оболочки гладкой компактной поверхности с границей и углами при $n \geqslant 2$.

ЗАмЕчАниЕ 5. Как видно из приведенных списков, граница выпуклых оболочек и граница зон локальной транзитивности в общем положении липшицева. По-видимому, этот факт верен в любой размерности.

ЗАмЕчАниЕ 6. Более того, во всех случаях справедливо следующее утверждение, которое доставляет пример высказанного в работе [19] принципа, что в ряде конструкций теории управления "хорошее превалирует", в отличие от типичного для теории особенностей принципа “хрупкости хорошего”, сформулированного В. И. Арнольдом [30].

Рассмотрим росток $K$ зоны транзитивности общего положения, базовая точка которого отвечает принадлежности начала координат $C^{1}$-гладкому ростку выпуклой оболочки. Тогда граница $\Sigma$ либо является гладкой, либо $K$ расположен в той (“большей") части ростка дополнения к $\Sigma$, которая ни в каких гладких локальных координатах не помещается внутри некоторого полупространства.

ЗАмЕчАНИЕ 7. Границы выпуклых оболочек кривых и поверхностей содержат развертывающиеся или плоские области. При диффеоморфизмах это свойство теряется: случаи различного расположения плоских частей и прямых на 
развертывающихся поверхностях могут отвечать диффеоморфным особенностям. Хотя в формулировках теорем 1-12 указана только грубая классификация с точностью до диффеоморфизмов, все различные случаи аффинной структуры особенностей можно получить методами, описанными ниже.

3.2. Преобразования Лежандра и опорные гиперплоскости. В этом пункте перечислены основные конструкции и утверждения (см. [25], [27]), на которых базируются доказательства теорем предыдущего пункта и которые могут быть полезны при исследовании особенностей во многих других задачах.

Сначала заметим, что если индикатриса задана как образ отображения $\mathbb{R}^{n} \rightarrow \mathbb{R}^{m}$ при $n \geqslant m, m=2,3$, то, согласно классическим результатам Р. Тома и Дж. Мазера о классификации особенностей типичных отображений (см., например, [17]), на границу выпуклой оболочки индикатрисы попадают в случае общего положения только точки гладкости видимого контура. Единственное исключение - это случай особенности “зонтик Уитни” $\mathbb{R}^{2} \rightarrow \mathbb{R}^{3}$. Во всех остальных случаях достаточно рассмотреть индикатрисы, являющиеся вложенными кривыми и поверхностями (возможно, с границами и углами), и их семейства.

Поверхности с краем и углами и кривые с концами удобно рассматривать единообразно, как специальные случаи стратифицированных вложенных подмногообразий в $\mathbb{R}^{3}$. Стратифицированным подмногообразием $J=\left\{I_{1}, \ldots, I_{s}\right\}$ будем называть набор замкнутых вложенных подмногообразий $I_{j}^{k_{j}}$ (стратов) размерностей $k_{j}$ таких, что имеется единственный страт наибольшей размерности и каждый страт меньшей размерности целиком принадлежит некоторому страту большей размерности. В случае индикатрис в трехмерном пространстве речь идет только об одномерных многообразиях $I^{1}$ с концевыми точками или о гладкой поверхности $I^{2}$ в $\mathbb{R}^{3}$, на которой задана гладкая кривая $I^{1}$ (край) или две трансверсально пересекающиеся кривые $I_{1}^{1}, I_{2}^{1}$ (угол), точку пересечения которых будем считать отдельным стратом. Однако описанные ниже конструкции легко переносятся и на многомерный случай.

Итак, в общем случае точке $q k$-мерного подмногообразия $I$ поставим в соответствие множество ростков в этой точке, диффеоморфное сфере $S^{3-k-1}$, всех коориентированных касательных плоскостей к подмногообразию в этой точке. Все такие ростки образуют гладкое лежандрово подмногообразие $L_{I} \approx$ $I \times S^{3-k-1}$ в пространстве $S T^{*} \mathbb{R}^{3}$ коориентированных контактных элементов. Проекция $\pi_{*}$ на пространство всех коориентируемых плоскостей этого подмногообразия, состоящая в забывании базовой точки ростка, является лежандровым отображением подмногообразия $L_{I}$ в двойственное пространство $\widehat{\mathbb{R}}^{3}$.

Образ (волновой фронт) $\hat{I}=\pi_{*}\left(L_{I}\right)$ этой проекции называется преобразованием Лежандра исходного подмногообразия $I$ или двойственной к $I$ поверхностью. Пусть $\hat{I}_{A}$ обозначает росток двойственной поверхности к $I$ на множестве всех касательных к $I$ плоскостей, проходящих через точку $A$.

Для стратифицированного подмногообразия $J$ преобразование Лежандра определяется как набор $\hat{J}$ преобразований Лежандра $\pi_{*}\left(L_{I_{j}}\right)$ для каждого страта $I_{j}$. Многообразие $J_{c}$ с краем или углом будем рассматривать как подмножество стратифицированного подмногообразия, заданное соответствующими неравенствами, поэтому в качестве $L_{J_{c}}$ будем брать подмножество касательных 
элементов в точках $J_{c}$. Преобразованием Лежандра $\hat{J}_{c}$ будут соответствующие подмножества двойственных поверхностей к стратам $J$.

Пространство всех коориентированных плоскостей в $\widehat{\mathbb{R}}^{3}$ расслоено над сферой нормалей с одномерным слоем (параллельных между собой плоскостей). Граница $H(\Gamma)$ выпуклой оболочки компактного подмножества Г определяется множеством $P(\Gamma)$ опорных плоскостей к $\Gamma$ : для каждого фиксированного направления нормали из всех параллельных между собой плоскостей, пересекающих эту оболочку, выбирается плоскость $P$ с максимальным значением координаты, градиент которой сонаправлен с положительной нормалью.

Отметим, что плоскость $P \in \widehat{\mathbb{R}}^{3}$ является опорной к $J$ тогда и только тогда, когда она является касательной к некоторым из стратов $I_{j}$ в одной или нескольких точках, т. е. принадлежит образу $\pi_{*} L_{J}$.

Для всякой опорной плоскости $P$ обозначим через $S_{P}=\gamma^{-1}(P)$ множество ее точек касания с $\Gamma$ и назовем его носителем $P$, а сами точки назовем базовыми. Число $\mu_{P}$ различных точек носителя $S_{P}$ будем называть кратностью.

Теорема трансверсальности Тома (см. [17]) для пространства мультиструй отображений или семейств отображений дает ограничения на возможные значения кратности опорных плоскостей, типы лежандровых отображений $\pi_{*}$ и т.д. Более того, принадлежность начала координат $O$ рассматриваемой опорной плоскости также налагает ограничения на возможные в общем положении типы особенностей отображения Лежандра.

Например, для поверхности Г общего положения мультиростки $\pi_{*} \mid S_{P}$ лежандрово устойчивы. Каждый росток лежандрово эквивалентен ростку либо с особенностью $A_{1}$ (поверхность в окрестности такой точки является графиком морсовской функции и ее квадратичная форма отрицательно определена), либо с особенностью $A_{3}$ (отвечающей лежандрову отображению графика функции $\left.h=x^{4}+\left(y-x^{2}\right)^{2}\right)$. Число точек в каждом носителе $S_{P}$ не превышает трех для особенностей $A_{1}$ и единицы для особенности $A_{3}$.

Таким образом, удается перечислить все возможные особенности двойственных поверхностей и классифицировать особенности выпуклых оболочек и зон транзитивности, используя следующие полезные свойства преобразования Лежандра.

1. Квадрат преобразования Лежандра типичной гиперповерхности - это тождественное преобразование. В самом деле, лежандрово подмногообразие имеет две лежандровы проекции: $\pi_{*}$ и стандартную проекцию расслоения $\pi$ : $S T^{*} \mathbb{R}^{3} \rightarrow \mathbb{R}$. Гладкое лежандрово подмногообразие $L_{I}$ однозначно восстанавливается по своему волновому фронту $\hat{I}$, если регулярные точки $\pi_{*}$ плотны в $L_{I}$ (см. [31]). Поэтому повторное преобразование Лежандра гиперповерхности $\hat{I}$ приводит к тому же лежандрову подмногообразию $L_{I}$ и проекции $\pi$, образ $\pi\left(L_{I}\right)$ которой совпадает с $I$.

2. Лежандрово подмногообразие $L_{I^{2}}$ гладкой поверхности $I^{2}$ регулярно проектируется на саму поверхность: в каждой точке касательная плоскость единственна. В окрестности выпуклой оболочки носителя $S_{P}$ опорной плоскости $P$ к $I^{2}$ граница выпуклой оболочки $H(I)$ задается опорными плоскостями, близкими к $P$. Росток двойственной поверхности $\left(\hat{I}^{2}, P\right)$, где $P$ - касательная 
к поверхности общего положения $I^{2}$ в точке $q$, является гладким, если $q$ не параболическая точка. В общей точке параболической линии $\delta$ этот росток диффеоморфен полукубическому цилиндру (т. е. бифуркационной диаграмме особенности $A_{2}$ функций). В изолированных точках линии $\delta$ преобразование Лежандра имеет особенность $A_{3}$ и росток $\left(\hat{I}^{2}, P\right)$ диффеоморфен ласточкиному хвосту. Точки типа $A_{2}$, не лежащие на краю, не лежат на выпуклой оболочке, так как касательная плоскость разделяет поверхность в окрестности $q$.

3. Лежандрово подмногообразие $L_{I^{1}}$ гладкой кривой $I^{1}$ состоит из окружностей $S_{q}$, составленных из касательных элементов к $I^{1}$ в ее точках $q$. Двойственная поверхность $\hat{I}^{1}$ является развертывающейся: в аффинной карте окружностям $S_{m}$ отвечают прямые. Множество опорных плоскостей $P$ к кривой, проходящих через точку $q$, образует связную замкнутую дугу $E_{q}$ на $S_{q}$. Опорную плоскость $P$, проходящую через $q \in I^{1}$, можно поворачивать вокруг касательной к кривой до тех пор, пока она не коснется кривой еще где-нибудь или не станет соприкасающейся плоскостью.

Росток выпуклой оболочки в окрестности точки $q$ одномерного страта $I^{1}$ задается ростком $\mathrm{Su}\left(I^{1}\right)$ на дуге $E_{q}$ опорных плоскостей, проходящих через $q$.

На кривой общего положения имеются отдельные точки простого уплощения (в окрестности такой точки в репере Френе кривая параметрически записывается как $\left.q_{1}=t, q_{2}=t^{2}+\cdots, q_{3}=t^{4}+\cdots\right)$, во всех остальных регулярных точках в системе координат Френе с началом в такой точке кривая имеет вид $q_{1}=t, q_{2}=t^{2}+\cdots, q_{3}=t^{3}+\cdots$. В окрестности соприкасающейся плоскости, заданной в обоих случаях уравнением $q_{3}=0$, двойственная поверхность имеет в случае уплощения особенность $A_{3}$ (ласточкин хвост), а в случае регулярной точки - особенность $A_{2}$ (полукубический цилиндр). Соприкасающаяся плоскость в регулярной неконцевой точке не может быть опорной: кривая лежит по разные стороны этой плоскости (в концевых точках соприкасающаяся плоскость может быть опорной).

4. Всякой точке $q \in \mathbb{R}^{3}$ отвечает плоскость $\hat{q}$ в двойственном пространстве, состоящая из всех плоскостей в $\mathbb{R}^{3}$, проходящих через q. Росток выпуклой оболочки в окрестности точки $q$, являющейся нульмерным стратом $J$ (вершиной угла, концевой точкой), определяется ростком $\mathrm{Su}(\hat{J})$ на выпуклой области $U_{q} \subset \hat{q}$, состоящей из всех опорных плоскостей, проходящих через $q$.

5. Преобразования Лежандра $\hat{I}_{1}$ и $\hat{I}_{2}$ двух стратов $I_{1} \subset I_{2}$ касаются вдоль своего пересечения $\hat{I}_{2} \cap \hat{I}_{1}$, состоящего из касательных плоскостей к $\hat{I}_{1}$ в точ$\operatorname{kax} \hat{I}_{2}$. В самом деле, поверхности $\hat{I}_{i}=\pi_{*}\left(L_{I_{i}}\right), i=1,2$, пересекаются по множеству общих касательных плоскостей в точках меньшего страта $I_{1}$. В силу инволютивности преобразования Лежандра касательные плоскости к $\pi_{*}\left(L_{I}\right)-$ это точки самого подмногообразия $I$. Поэтому касательные плоскости к $\hat{I}_{1}, \hat{I}_{2}$ в точках их пересечения отвечают общим точкам $I_{1} \cap I_{2}$ и, значит, совпадают, что и требовалось. Если на направлениях в $I_{2}$, трансверсальных к $I_{1}$, квадратичная форма подмногообразия $\hat{I}_{2}$ невырождена, то касание $\hat{I}_{1}$ и $\hat{I}_{2}$ имеет первый порядок.

6. Точке $Q \in \mathbb{R}^{3}$ отвечает плоскость $\widehat{Q}$ в двойственном пространстве, состоящая из всех плоскостей в $\mathbb{R}^{3}$, проходящих через $Q$. 
Точка $Q$ принадлежит границе выпуклой оболочки подмногообразия $B$ тогда и только тогда, когда $\widehat{Q}$ является опорной для подмножества $\operatorname{Su}(B)$ опорных плоскостей к $B$, т. е. открытое отрицательное полупространство $\widehat{Q}$ не содержит точек $\mathrm{Su}(B)$, а сама плоскость содержит такие точки. В частности, точка $O$ принадлежит выпуклой поверхности $X \subset \mathbb{R}^{3}$ тогда и только тогда, когда двойственная поверхность $\widehat{X}$ касается $\widehat{O}$.

7. Если $O$ принадлежит выпуклой оболочке носителя опорной плоскости некоторого трехпараметрического семейства подмногообразий $B$ размерности $k$, то в случае общего положения особенности преобразования Лежандра в каждой точке носителя являются особенностями общего положения преобразований Лежандра $k$-мерных подмногообразий. В самом деле, размерность выпуклой оболочки носителя из $l$ точек не превосходит $l-1$. Для того чтобы $O$ принадлежала выпуклой оболочке носителя, необходимо выполнение не менее $3-l+1$ условий. Для того чтобы $l$ точек попало на соприкасающуюся плоскость, нужно $l$ условий. Для того чтобы вырожденная точка появилась на носителе, нужно не менее одного условия. Таким образом, в этом случае необходимо выполнение более трех независимых условий, что невозможно в общем положении при трех параметрах.

Классификация типичных особенностей границы зоны транзитивности получается теперь перечислением всех возможных расположений нуля в носителе опорной плоскости, описанием соответствующих семейств двойственных поверхностей и их опорных подмножеств и, наконец, применением следующих результатов из теории особенностей.

Диффеоморфизм $\Phi: \widehat{\mathbb{R}}^{3} \rightarrow \widehat{\mathbb{R}}^{3}$, переводящий плоскость $\widehat{O}$ в себя и сохраняющий ее положительное полупространство, называется допустимым. Если $\Phi$ переводит одно в другое преобразования Лежандра $\mathrm{Su}\left(J_{i}\right), i=1,2$, двух стратифицированных многообразий, то очевидно, что точка $O$ принадлежит границе $H\left(J_{1}\right)$, тогда и только тогда, когда она принадлежит $H\left(J_{2}\right)$. Семейство допустимых диффеоморфизмов $\Phi_{m}$, расслоенное над диффеоморфизмом пространства параметров, действуя на наборы поверхностей $\hat{I}_{j}$, зависящие от параметров, переводит одно в другое соответствующие зоны транзитивности. Приводя одновременно к нормальному виду уравнения поверхностей $\hat{I}_{j}$ с помощью семейств допустимых диффеоморфизмов, получим нормальные формы зоны транзитивности. Для этого удобно рассматривать действие, $\Phi_{m}$ как контактного преобразования произведения уравнений всех компонент (см. [32]).

Обозначим через $P_{i}(x), i=0, \ldots, n,-$ многочлены степени $k_{i}$ от $x \in \mathbb{R}$ вида $P_{0}=x^{k_{0}}+\sum_{j=0}^{k_{0}-2} x^{j} a_{0 j}, P_{i}=x^{k_{i}}+\sum_{j=0}^{k_{i}-1} x^{j} a_{i j}, i=1, \ldots, n$. Обозначим через $a \in \mathbb{R}^{N}$ вектор коэффициентов всех этих многочленов. Заметим, что $P_{0}$ является стандартной миниверсальной деформацией особенности $A_{k_{0}-1}$, а $P_{i}$ при $i=1, \ldots, n$ является версальной деформацией особенности $A_{k_{i}-1}$.

Лемма. Пусть $g_{i}(x, b), i=0, \ldots, n,-$-екоторье функиии от $x$ с параметрами $b \in \mathbb{R}^{N}$ такие, что значения и все производные функиии $g_{i}(x, 0)$ до порядка $k_{i}-1$ в нуле обращаются в нуль $\left(g_{i}(x, 0)\right.$ имеет особенность $\left.A_{k_{i}-1}\right)$, и пусть 
$g(x, b)=\prod_{i=0}^{n} g_{i}(x, b)$. Тогда существует контактная эквивалентность, состоящая из диффеоморфизма $(x, b) \mapsto(X(x, b), B(b))$ и ненулевой функиии $\varphi(x, b)$, приводящая произведение $g \kappa$ виду $g(x, b)=\varphi(x, b) \prod_{i=0}^{n} P_{i}(X, B)$.

Утверждение леммы равносильно версальности описанного ниже отображения относительно специальной группы отображений. Рассмотрим отображение $G: \mathbb{R}^{k} \rightarrow \mathbb{R}^{n+1}, G: x \mapsto\left(g_{0}(x), \ldots, g_{n}(x)\right)$. В пространстве-образе рассмотрим набор $Y$ координатных гиперплоскостей $Y=\bigcup\left\{y_{i}=0\right\}$. Пусть $D$ - группа диффеоморфизмов $\mathbb{R}^{n+1}$, сохраняющих $Y$, т. е. имеющих вид $\theta:\left(y_{0}, \ldots, y_{n}\right) \mapsto$ $\left(h_{0}(y) y_{0}, \ldots, h_{n}(y) y_{n}\right)$. Два отображения $G_{1}$ и $G_{2}$ назовем $Y$-контактно эквивалентными, если существует семейство, зависящее от $x$, диффеоморфизмов $\theta_{x}$ из $D$ такое, что $G_{2}(x)=\theta_{x} \circ G_{1}(X(x))$ для некоторой замены переменной $x \mapsto X(x)$.

Для семейства $G_{m}: \mathbb{R}^{k} \times \mathbb{R}^{N} \rightarrow \mathbb{R}^{n+1}$ отображений $G$, зависящих от параметров $а$ из $\mathbb{R}^{N}$, естественно определяется параметрическая контактная эквивалентность: параметры $a$ заменяются на параметры $b$ с помощью диффеоморфизма; на переменные $x$ действует диффеоморфизм вида $x \mapsto X(x, a)$; в образе $G_{m}$ действует семейство диффеоморфизмов $\theta_{x, a}: \mathbb{R}^{n+1} \rightarrow \mathbb{R}^{n+1}$. Естественным образом вводятся понятия версальной и инфинитезимально версальной деформации отображения $G$. Легко видеть, что для указанной группы преобразований $D$ верен аналог теоремы версальности, поскольку она является геометрической в смысле Дж. Дэймона.

Заметим, что отображение $\widetilde{P}:(x, a) \mapsto\left(P_{0}, \ldots, P_{n}\right)$ является инфинитези-

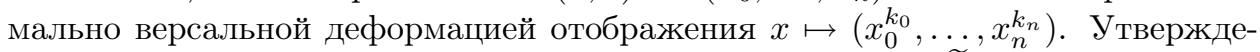
ние леммы вытекает теперь из версальности отображения $\widetilde{P}$. При эквивалентности из группы $D$ каждая компонента умножается на ненулевой множитель, значит, и произведение умножается на ненулевой множитель.

\section{4. Особенности нелокальной управляемости}

Здесь мы обсуждаем сначала гёльдеровость границы достижимости типичной гладкой системы на гладком многообразии, а затем для гладких систем на поверхностях типичные особенности границы этого множества и зон нелокальной транзитивности.

4.1. Гёльдеровость границы достижимости. Стартовым называется подмножество фазового пространства, с которого можно начинать допустимое движение. Множество точек, в каждую из которых допустимым движением система может быть переведена из некоторой точки стартового подмножества за конечное время, называется положительной орбитой этого подмножества или множеством достижимости. Если же зафиксировано целевое подмножество, на которое нужно привести систему, то мы приходим к аналогичному понятию отрицательной орбиты или множества управляемости, доставляющего множество точек, из каждой из которых такое приведение возможно. 
Исследование множеств достижимости (управляемости) системы обычно предшествует оптимизации ее траекторий и является важной прикладной задачей, поэтому такие исследования, а также внутренние и внешние оценки множеств достижимости проводились и проводятся различными методами (см., например, [19], [33]-[36] и библиографию в этих работах).

Теорема 13 [13]. Для типичной управляемой системы и любого стартового множества граница множества достижимости либо пуста, либо является локально липшицевой или гёльдеровой гиперповерхностью в фазовом пространстве.

Доказательство этой теоремы основано на оценке "снизу" ростков положительных и отрицательных орбит точек при малых временах, непрерывной по точкам фазового пространства. Суть этого доказательства в следующем: в гладких локальных координатах вблизи точки границы достижимости строятся непрерывные поля открытых конусов $K^{+}$и $K^{-}, K^{-}=-K^{+}$, значения которых в точке $P$ содержатся в ее положительной и отрицательной орбите соответственно (см., например, [13], [19], [33]).

Отсюда следует, что для точки $P$ границы достижимости вблизи этой точки в конусе $K^{-}(P)$ нет точек достижимости, а конус $K^{+}(P)$ целиком лежит в множестве достижимости. Следовательно, объединение этих точек определяет поверхность, локально липшицеву или гёльдерову, если построенное поле конусов позволяет дать соответственно локальную липшицеву или гёльдерову оценку для этой поверхности (рис. 13).

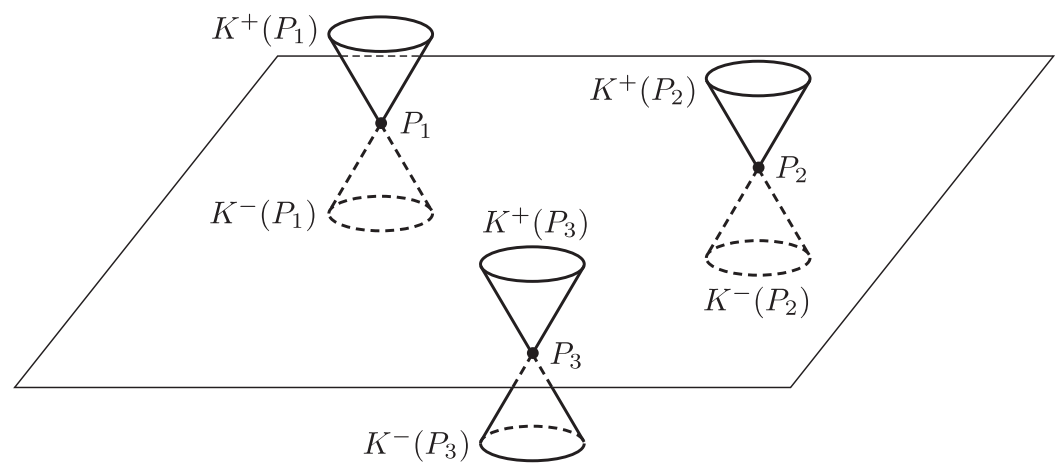

Рис. 13. Гёльдеровость границы достижимости

\section{2. Особенности нелокальной управляемости в двумерном случае.}

Зоной нелокальной транзитивности управляемой системы называется подмножество в фазовом пространстве, совпадающее с пересечением отрицательной и положительной орбиты любой из своих точек. Для типичных систем любая ее зона нелокальной транзитивности открыта.

Например, для рассмотренной выше бидинамической системы на плоскости $\mathbb{R}_{x, y}^{2}$ с полями скоростей $(1,0)$ и $(x-y, x+y)$ вся плоскость является зоной нелокальной транзитивности. 
Заметим, что, начиная движение из внутренней точки зоны нелокальной транзитивности, можно перейти в любое другое состояние из зоны и вернуться обратно за конечное время с помощью допустимого движения. Ясно, что связная компонента зоны локальной транзитивности (за малое время) целиком лежит в некоторой зоне нелокальной транзитивности.

Для модели пловца в потоке жидкости при $\beta>2$, рассмотренной выше, отрицательная орбита любой точки зоны локальной транзитивности (за малое время) совпадает со всей плоскостью, а положительная орбита является открытой областью, ограниченной объединением сложенных особых точек уравнения (1) и тех сепаратрис сложенных седел, что входят в сложенные узлы. Таким образом, здесь положительная орбита является также и зоной нелокальной транзитивности.

Учитывая, что поле предельных направлений допустимых скоростей гладко вне замыкания зоны локальной транзитивности, получаем, что граница этой зоны гладка всюду, кроме, возможно, особых точек типа "сложенный узел". Дело в том, что показатели этих узлов (а именно, отношение наибольшего по модулю собственного числа линеаризации поля в особой точке к наименьшему) равны $\beta-1$, а граница зоны нелокальной транзитивности вблизи каждого из них содержит две фазовые кривые, входящие в узел с противоположных направлений. Следовательно, если этот показатель - нецелое число, а эти кривые неособые кривые этого узла (т. е. не те, что продолжаются гладко через особую точку), то эта граница не является гладкой в этом узле точно так же, как и в случае обычного узла с нецелым показателем. Для сложенного узла, лежащего на границе достижимости или границе зоны нелокальной транзитивности, такой картины можно добиться малым шевелением типичного неравенства (управляемой системы) вблизи этого узла [19], [24].

При потоке воды $\left(x^{2}+(\beta y)^{2}, 0\right), \beta>0$, в модели пловца поле предельных направлений системы определено в области $x^{2}+(\beta y)^{2} \geqslant 1$ и трансверсально границе этой области всюду, за исключением точек $( \pm 1,0)$, где оно имеет особые точки типа "сложенное седло". Зона локальной транзитивности (за малое время) состоит из дополнения к этой области, а также ее границы, за исключением этих двух особых точек. Для любой из точек этой зоны ее положительная орбита расположена в точности справа от объединения особой точки $(-1,0)$ с выходящими из нее сепаратрисами, а отрицательная орбита - в точности слева от объединения особой точки $(1,0)$ с входящими в нее сепаратрисами. Таким образом, зона нелокальной транзитивности представляет собой линзу, граница которой гладка всюду, за исключением точек пересечения этих сепаратрис, в которых зона нелокальной транзитивности имеет углы (рис. 14). Нетрудно видеть, что при достаточно малом изменении поля сноса граница этой линзы сохранит свою структуру, хотя ее расположение может немного измениться, в частности, эти углы на ней, возможно, немного сместятся.

Точку границы зоны нелокальной транзитивности будем называть особой mочкой muna $p, 1 \leqslant p \leqslant 6$, если росток этой зоны в этой точке $C^{\infty}$-диффеоморфен ростку в нуле множества

1) $y>|x|$ либо $y<|x|$ при $p=1$;

2) $y>x^{p-1}|x|$ при $p=2,3$; 


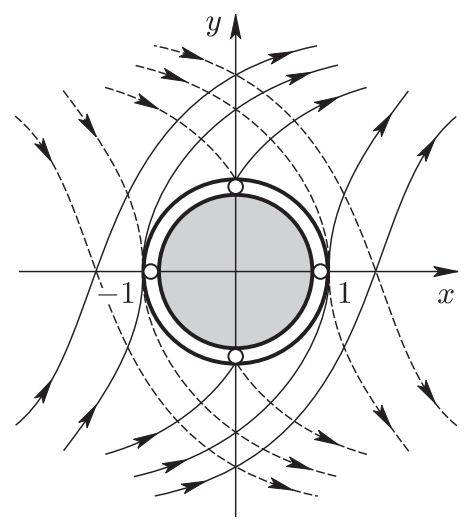

Рис. 14. Углы на границе зоны нелокальной транзитивности

3) $\varepsilon x^{\alpha}<y<x$, где $\varepsilon= \pm 1$, при $p=4$;

4) $y<(\varepsilon+c(\operatorname{sgn} x-1))|x|^{\alpha}$, где $\varepsilon= \pm 1, \varepsilon / 2 \neq c \in \mathbb{R}$ при $p=5$;

5) $y<h(x)$ при $p=6$, где график $y=h(x)$ вблизи нуля есть замыкания двух неособых фазовых кривых сложенного узла уравнения

$$
y=\left(\frac{d y}{d x}+\frac{\alpha}{(\alpha+1)^{2}} \frac{x}{2}\right)^{2},
$$

входящих в нуль с противоположных направлений.

Всюду здесь $\alpha>1$ (нецелое число) - показатель соответствующего (сложенного) узла.

Для гладких управляемых систем на компактных ориентируемых поверхностях (без края) с гладким компактным многообразием в качестве множества значений управляющего параметра (или дизъюнктным объединением таковых) справедлива следующая теорема.

Теорема 14. Граница зоны нелокальной транзитивности типичной системы либо пуста, либо является гладким одномерным подмногообразием с особъми точками типов 1)-6). Более того, для типичной системы и любой системы, достаточно $C^{4}$-близкой $\kappa$ ней, зонъ нелокальной транзитивности этих систем переводятся одни в другие дифбеоморфизмом фазового пространства, $C^{0}$-близким к тождественному.

Аналогичная теорема справедлива и для множества достижимости при фиксированном гладком подмногообразии в качестве стартового множества, где дополнительно у границы достижимости могут появиться точки негладкости первых двух типов в точках стартового множества, попадающих на эту границу. Доказательство этих результатов есть в [19], там же приведено и полное описание природы этих особенностей. Это доказательство основано на методах теории особенностей дифференцируемых отображений [17] и качественной теории динамических систем [37]. Первые из них используются для анализа локальных особенностей полей предельных направлений допустимых скоростей 
системы, доказательства их устойчивости, а также непрерывной зависимости поля от типичной системы, а вторые применяются для анализа в целом поведения сети интегральных кривых этого поля, как и в случае обычных полей направлений и векторных полей.

ЗАмЕчАНиЕ 8. При наличии на границе множества достижимости либо на границе зоны нелокальной транзитивности особых точек четвертого и пятого типов, возникающих в узлах допустимых полей скоростей, соответствующих изолированным значениям управляющего параметра, и типа 6 , наблюдаемых в сложенных узлах, можно гарантировать, вообще говоря, соответственно лишь $C^{1}$ - и $C^{2}$-гладкость переводящего диффеоморфизма в точках границы с такими особенностями.

ЗАмЕчАниЕ 9. Для простейших динамических неравенств классификация особенностей зон нелокальной транзитивности получена в [24].

\section{3. Структурная устойчивость управляемых систем на поверхно-} стях. Понятие структурного устойчивого (грубого) векторного поля было введено А. А. Андроновым и Л. С. Понтрягиным в 1937 г. [38] для полей на диске, а затем было распространено на динамические системы на многообразиях. Для управляемых систем оно вводится аналогично, только роль траекторий играют положительные и отрицательные орбиты точек: управляемая система называется структурно устойчивой, если положительные и отрицательные орбиты точек любой достаточно близкой к ней системы переводятся в такие же орбиты первоначальной системы гомеоморфизмом фазового пространства, близким к тождественному.

Легко построить пример структурно устойчивой управляемой системы такой будет, например, бидинамическая система на окружности, задаваемая двумя дифференцируемыми полями скоростей с невырожденными попарно различными особыми точками, структурно устойчива. Действительно, после выбора ориентации окружности эти особые точки разобьют окружность на интервалы, на которых обе скорости либо положительны, либо отрицательны, либо имеют противоположные знаки. Для любой точки ее положительная орбита либо совпадет со всей окружностью, либо будет начинаться в ближайшей против хода движения точке, перед которой обе скорости положительны, и заканчиваться в ближайшей по ходу движения точке, после которой обе скорости становятся отрицательными. Для отрицательной орбиты аналогично, только знаки скоростей нужно сменить на противоположные.

В силу предположения о невырожденности особых точек и их попарного различия для бидинамической системы, достаточно близкой к исходной в $C^{1}$-топологии, особые точки ее допустимых полей скоростей мало изменятся и будут близки к соответствующим особым точкам исходной системы, и, в частности, порядок их расположения на окружности будет прежним.

Следовательно, взяв гомеоморфизм окружности, переводящий соответствующие особые точки полей одни в другие и, например, линейный на интервалах между этими точками, мы получим гомеоморфизм окружности, переводящий положительные и отрицательные орбиты точек окружности одной из этих 
систем в такие же орбиты другой. Таким образом, гладкая бидинамическая система на окружности с полями скоростей, имеющими попарно различные невырожденные особые точки, является структурно устойчивой.

ТЕОРЕма 15. Типичная гладкая управляемая система на окружности с компактным гладким многообразием (или дизгюнктным оббединением таковых) в качестве пространства управлений структурно устойчива.

Для доказательства этой теоремы достаточно заметить, что для типичной системы на окружности ее минимальная и максимальная скорости движения являются гладкими функциями с устойчивыми особенностями типа $y=-|x|$ и $y=|x|$ соответственно (см. [10], [17]), не лежащими на нулевом уровне соответствующей скорости. Следовательно, для доказательства структурной устойчивости такой системы можно применить те же рассуждения, что и в случае бидинамической системы выше.

Как и случае векторных полей, аналогичный результат справедлив и для систем на поверхностях.

ТЕОРема 16 [39]. Типичная гладкая управляемая система на замкнутой ориентируемой поверхности с компактным гладким многообразием или дизбюнктным оббединением таковых в качестве пространства управлений, имеющим не менее двух различных точек, структурно устойчива.

Таким образом, по отношению к структурной устойчивости типичные управляемые системы ведут себя так же, как типичные векторные поля на компактной ориентируемой поверхности [37].

Конечно, структурно устойчивые управляемые системы есть на многообразиях любой размерности. Например, на компактном гладком связном многообразии типичная полностью управляемая система (т. е. когда и положительная и отрицательная орбита любой точки совпадает со всем многообразием) структурно устойчива, что очевидно. Однако нетривиальные примеры структурно устойчивых систем на многообразиях размерности большей двух нам не известны.

Подмножество фазового пространства называется устойчивым, если для любой его окрестности существует другая его окрестность такая, что каждое допустимое движение системы с начальной точкой из последней окрестности продолжается на все последующие времена и при любом своем продолжении не покидает первой окрестности. Если к тому же для любой последовательности окрестностей $U_{1} \supset U_{2} \supset \cdots \supset U_{n} \supset \cdots$ этого множества любое продолженное движение происходит в $U_{n}$, начиная с некоторого момента времени, то это подмножество называется асимптотически устойчивым [40].

ТЕОрема 17 [39]. Для типичной гладкой управляемой системы на замкнутой ориентируемой поверхности с компактным гладким многообразием (или дизгюнктным обгединением таковых) в качестве пространства управлений, имеющим не менее двух различных точек, положительная орбита точки асимптотически устойчива, если эта точка принадлежит внутренности этой орбиты. 
Таким образом, если управляемый объект находился достаточно близко к такой орбите, то с течением времени он только будет приближаться к ней.

Следующий пример иллюстрирует последние две теоремы. Рассмотрим бидинамическую систему на сфере с двумя структурно устойчивыми векторными полями. Одно допустимое поле скоростей имеет невырожденный устойчивый узел на южном полюсе и неустойчивый невырожденный узел на северном. Остальные фазовые кривые этого поля стекают с северного полюса на южный и являются меридианами вне достаточно малых окрестностей этих полюсов.

Второе допустимое поле скоростей имеет два простых цикла, являющихся параллелями и расположенных чуть севернее и чуть южнее экватора. Северный цикл неустойчив; фазовая кривая, сматывающаяся с него, либо наматывается на южный цикл, либо входит в невырожденный устойчивый фокус вблизи северного полюса. Южный цикл устойчив; фазовая кривая, наматывающаяся на него, либо сматывается с северного цикла, либо выходит из невырожденного неустойчивого фокуса вблизи южного полюса (рис. 15).

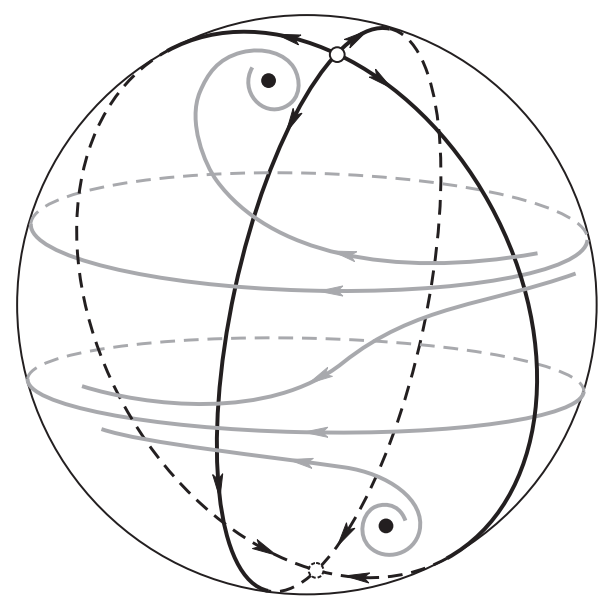

Рис. 15. Структурно устойчивая система на сфере

Эта управляемая система имеет две зоны нелокальной транзитивности: северную и южную. Они расположены соответственно выше северного и ниже южного циклов, а между ними находится экваториальное кольцо, в котором фазовые кривые одного поля допустимых скоростей являются меридианами, а фазовые кривые другого поля сматываются с северного цикла и наматываются на южный цикл.

Для точки северной (соответственно южной) зоны нелокальной транзитивности отрицательная (соответственно положительная) орбита совпадает с этой зоной, а положительная (соответственно отрицательная) - со всей сферой. Для точки границы этой зоны одна орбита совпадает с замыканием этой зоны, а другая - с замыканием дополнения к этой зоне.

Рассмотрим внутреннюю точку экваториального кольца. Проведем через нее фазовые кривые полей допустимых скоростей и возьмем по два участка 
положительных и отрицательных полутраекторий этих кривых: участки, заключенные между нашей точкой и двумя ближайшими к ней точками пересечения этих кривых. Положительная (соответственно отрицательная) орбита нашей точки расположена под (соответственно над) объединением двух из этих четырех участков, принадлежащих положительным (соответственно отрицательным) полутраекториям.

Понятно, что наблюдаемая в этом примере структура орбит точек будет такой же и для любой достаточно близкой в $C^{k}$-топологии управляемой системы при $k \geqslant 1$; гомеоморфизмом сферы, близким к тождественному, орбиты точек одной из этих систем переводятся в орбиты точек другой системы. Таким образом, рассмотренная управляемая система является структурно устойчивой.

\section{Список литературы}

[1] С. Смейл, "Глобальный анализ и экономика, I. Оптимум Парето и обобщение теории Морса", УМH, 27:3(165) (1972), 177-187; пер. с англ.: S. Smale, "Global analysis and economics. I. Pareto optimum and a generalization of Morse theory", Dynamical systems, Proc. Sympos. (Univ. Bahia, Salvador, 1971), Academic Press, New York, 1973, 531-544.

[2] В. И. Арнольд, Задачи Арнольда, Фазис, М., 2000, х+452 с.; англ. пер.: V. I. Arnold, Arnold's problems, Springer-Verlag, Berlin; Phasis, Moscow, 2005, 640 pp.

[3] V.I. Arnol'd, "Wave front evolution and equivariant Morse lemma", Comm. Pure and Appl. Math., 29:6 (1976), 557-582.

[4] В. М. Закалюкин, "Перестройки волновых фронтов, зависящих от одного параметра", Функи. анализ и его прил., 10:2 (1976), 69-70; англ. пер.: V. M. Zakalyukin, "Reconstructions of wave fronts depending on one parameter", Funct. Anal. Appl., 10:2 (1976), 139-140.

[5] В. М. Закалюкин, "Особенности выпуклых оболочек гладких многообразий", Функи. анализ и его прил., 11:3 (1977), 76-77; англ. пер.: V. M. Zakalyukin, "Singularities of convex hulls of smooth manifolds", Funct. Anal. Appl., 11:3 (1977), 225-227.

[6] В. Д. Седых, “Особенности выпуклой оболочки кривой в $\mathbb{R}^{3}$ ”, Функи. анализ и его прил., 11:1 (1977), 81-82; англ. пер.: V. D. Sedykh, "Singularities of the convex hull of a curve in $\mathbb{R}^{3 ",}$ Funct. Anal. Appl., 11:1 (1977), 72-73.

[7] В. Д. Седых, "Модули особенностей выпуклых оболочек", УМH, 36:5(221) (1981), 191-192; англ. пер.: V.D. Sedykh, "Moduli of singularities of convex hulls", Russian Math. Surveys, 36:5 (1981), 175-176.

[8] В. Д. Седых, "Строение выпуклой оболочки пространственной кривой", Тр. семин. им. И. Г. Петровского, 6, 1981, 239-256; англ. пер.: V. D. Sedykh, "Structure of the convex hull of a space curve", J. Sov. Math., 33 (1986), 1140-1153.

[9] В. Д. Седых, "Функциональные модули особенностей выпуклых оболочек многообразий коразмерностей 1 и 2", Матем. сб., 119(161):2(10) (1982), 233-247; англ. пер.: V.D. Sedykh, "Functional moduli of singularities of convex hulls of manifolds of codimensions 1 and 2", Math. USSR-Sb., 47:1 (1984), 223-236.

[10] Л.Н. Брызгалова, "Особенности максимума функции, зависящей от параметров”, Функи. анализ и его прил., 11:1 (1977), 59-60; англ. пер.: L. N. Bryzgalova, "Singularities of the maximum of a parametrically dependent function", Funct. Anal. Appl., 11:1 (1977), 49-51.

[11] Л.Н. Брызгалова, "О функциях максимума семейства функций, зависящих от параметров”, Функи. анализ и его прил., 12:1 (1978), 66-67; англ. пер.: 
L. N. Bryzgalova, "Maximum functions of a family of functions depending on parameters", Funct. Anal. Appl., 12:1 (1978), 50-51.

[12] В. И. Матов, "Топологическая классификация ростков функций максимума и минимакса семейств функций общего положения", УМH, 37:4(226) (1982), 167-168; англ. пер.: V.I. Matov, "The topological classification of germs of the maximum and minimax functions of a family of functions in general position", Russian Math. Surveys, 37:4 (1982), 127-128.

[13] А. А. Давыдов, “Квазигельдеровость границы достижимости”, Тр. семин. по тензорному и векторному анализу, 22, Изд-во МГУ, М., 1985, 25-30; англ. пер.: A. A. Davydov, "The quasi-Hölder property of the boundary of attainability", Selecta Math. Soviet, 9:3 (1990), 229-234.

[14] B. Jakubczyk, F. Przytycki, Singularities of k-tuples of vector fields, Diss. Math. (Rozprawy Mat.), 213, Państwowe Wydawn. Naukowe, Warszawa, 1984, 64 pp.

[15] Р. Айзекс, Дифференциальные игры, Мир, М., 1967, 480 с.; пер. с англ.: R. Isaacs, Differential games. A mathematical theory with applications to warfare and pursuit, control and optimization, Wiley, New York-London-Sydney, 1965, xvii+384 pp.

[16] Л. С. Понтрягин, В.Г. Болтянский, Р. В. Гамкрелидзе, Е.Ф. Мищенко, Математическая теория оптимальных прочессов, Физматгиз, М., 1961; англ. пер.: L. S. Pontryagin; V. G. Boltyanskii, R. V. Gamkrelidze, E. F. Mishchenko, The mathematical theory of optimal processes, Wiley, New York-London, 1962, viii+360 pp.

[17] В. И. Арнольд, А. Н. Варченко, С. М. Гусейн-Заде, Особенности дифферениируемых отображений, т. I, Наука, M., 1982, 304 с.; англ. пер.: V. I. Arnol'd, S. M. Gusern-Zade, A. N. Varchenko, Singularities of differentiable maps, v. I: The classification of critical points, caustics and wave fronts, Monogr. Math., 82, Birkhäuser, Boston, MA, 1985, xi+382 pp.

[18] Р. Калман, П. Фалб, М. Арбиб, Очерки по математической теории систем, 2-е изд., УРСС, М., 2004, 398 с.; пер. с англ.: R. E. Kalman, P. L. Falb, M. A. Arbib, Topics in mathematical system theory, McGraw-Hill, New York-Toronto, ON-London, 1969, xiv +358 pp.

[19] A. A. Davydov, Qualitative theory of control systems, Transl. Math. Monogr., 141, Amer. Math. Soc., Providence, RI, 1994, viii+147 pp.

[20] А. Д. Мышкис, "О дифференциальных неравенствах с локально ограниченными производными", Ученые записки Харъковского государственного ун-та им. A.M. Горъкого, CXXXVIII: Ученые записки механико-математического факультета и Харьковского математического общества, сер. 4, т. 30, ХГУ им. А. М. Горького, Харьков, 1964, 152-163.

[21] Н.Н. Петров, "Об управляемости автономных систем", Дифферени. уравнения, 4:4 (1968), 606-617; англ. пер.: N. N. Petrov, "Controllability of autonomous systems", Differ. Equ., 4 (1972), 311-317.

[22] Н. Н. Петров, “Локальная управляемость автономных систем”, Дифферени. уравнения, 4:7 (1968), 1218-1232; англ. пер.: N. N. Petrov, "Local controllability of autonomous systems", Differ. Equ., 4 (1972), 632-639.

[23] А. А. Давыдов, “Локальная управляемость типичных динамических неравенств на поверхностях", Особенности гладких отображений с дополнительными структурами, Сборник статей, Тр. МИАН, 209, Наука, Физматлит, М., 1995, 84-123; англ. пер.: A. A. Davydov, "Local controllability of typical dynamical inequalities on surfaces", Proc. Steklov Inst. Math., 209 (1995), 73-106.

[24] Ю. А. Гришина, А. А. Давыдов, “Структурная устойчивость простейших динамических неравенств", Динамические системы и оптимизащия, Сборник статей. K 70-летию со дня рождения академика Дмитрия Викторовича Аносова, Тр. МИАН, 256, Наука, М., 2007, 89-101; англ. пер.: Yu. A. Grishina, А. A. Davydov, 
"Structural stability of simplest dynamical inequalities", Proc. Steklov Inst. Math., 256 (2007), 80-91.

[25] В.М. Закалюкин, А.Н. Курбацкий, "Особенности огибающих семейств плоскостей в теории управления", Оптимальное управление, Сборник статей. K 60-летию со дня рождения профессора Виктора Ивановича Благодатских, Тр. МИАН, 262, МАИК, М., 2008, 73-86; англ. пер.: V. M. Zakalyukin, А. N. Kurbatskii, "Envelope singularities of families of planes in control theory", Proc. Steklov Inst. Math., 262 (2008), 66-79.

[26] В. М. Закалюкин, А. Н. Курбацкий, "Выпуклые оболочки кривых и особенности множества транзитивности в $\mathbb{R}^{3 "}$, Современные проблемы математики и механики, 4, 2, Изд-во Моск. ун-та, М., 2009, 3-23.

[27] В. М. Закалюкин, А. Н. Курбацкий, "Выпуклые оболочки поверхностей с краем и углами и особенности зоны транзитивности в $\mathbb{R}^{3}$ ", Дифференииалъные уравнения u топология. I, Сборник статей. K 100-летию со дня рождения академика Льва Семеновича Понтрягина, Тр. МИАН, 268, МАИК, М., 2010, 284-303; англ. пер.: V. M. Zakalyukin, A. N. Kurbatskii, "Convex hulls of surfaces with boundaries and corners and singularities of transitivity zone in $\mathbb{R}^{3}$ ", Proc. Steklov Inst. Math., 268 (2010), 274-293.

[28] А. Н. Курбацкий, "Особенности зоны транзитивности поверхностей с краем в $\mathbb{R}^{3}$ ”, УМH, 65:3(393) (2010), 199-200; англ. пер.: A. N. Kurbatskii, "Singularities of the

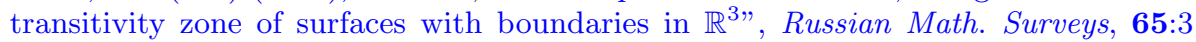
(2010), 583-585.

[29] В.М. Закалюкин, "Особенности выпуклых оболочек гладких многообразий", Функи. анализ и его прил., 11:3 (1977), 76-77; англ. пер.: V. M. Zakalyukin, "Singularities of convex hulls of smooth manifolds", Funct. Anal. Appl., 11:3 (1977), $225-227$.

[30] В. И. Арнольд, Теория катастроф, 3-е изд., Наука, М., 1990, 128 с.; англ. пер. 2-го изд.: V. I. Arnold, Catastrophe theory, Springer-Verlag, Berlin, 1984, iv+79 pp.

[31] В. М. Закалюкин, "Перестройки фронтов, каустик, зависящих от параметра, версальность отображении", Итоги науки и техники. Соврем. пробл. матем., 22, ВИНИТИ, М., 1983, 56-93; англ. пер.: V. M. Zakalyukin, "Reconstructions of fronts and caustics depending on a parameter, and versality of mappings", J. Sov. Math., 27:3 (1984), 2713-2735.

[32] В. В. Горюнов, В. М. Закалюкин, "Об устойчивости проекций лагранжевых многообразий с особенностями”, Функи. анализ и его прил., 38:4 (2004), 13-21; англ. пер.: V.V. Goryunov, V. M. Zakalyukin, "On stability of projections of Lagrangian varieties", Funct. Anal. Appl., 38:4 (2004), 249-255.

[33] А. М. Вершик, В.Я. Гершкович, "Неголономные динамические системы. Геометрия распределений и вариационные задачи", Динамические системы - 7, Итоги науки и техники. Соврем. пробл. матем. Фундам. напр., 16, ВИНИТИ, M., 1987, 5-85; англ. пер.: A. M. Vershik, V. Ya. Gershkovich, "Nonholonomic dynamical systems, geometry of distributions and variational problems", Dynamical systems. VII, Encycl. Math. Sci., 16, Springer, Berlin, 1994, 1-81.

[34] П. Варайя, А.Б. Куржанский, "О проблеме достижимости при постоянно действующих возмущениях", Докл. РАН, 372:4 (2000), 446-450; англ. пер.: P. Varaiya, A. B. Kurzhanskij, "The reachability problem under constant perturbations", Dokl. Math., 61:3 (2000), 380-384.

[35] Т. Ф. Филиппова, "Дифференциальные уравнения эллипсоидальных оценок множеств достижимости нелинейной динамической управляемой системы", Тр. ИММ УрО РАН, 16, 1, Изд-во ИММ УрО РАН, Екатеринбург, 2010, 223-232; англ. пер.: T. F. Filippova, "Differential equations of ellipsoidal estimates for reachable sets of a nonlinear dynamical control system", Proc. Steklov Inst. Math., 271, suppl. 1 (2010), 75-84. 
[36] Ф.Л. Черноусько, Ои,енвание фазового состояния динамических систем, Наука, М., 1988, 320 с.

[37] Ж. Палис, В. Ди Мелу, Геометрическал теория динамических систем, УРСС, M., 1998, 300 с.; пер. с англ.: J. Palis (Jr.), W. de Melo, Geometric theory of dynamical systems. An introduction, Springer-Verlag, New York-Berlin, 1982, xii+198 pp.

[38] А.А. Андронов, Л. С. Понтрягин, "Грубые системы", Докл. АН СССР, 14:5 (1937), 247-250.

[39] А.А. Давыдов, "Структурная устойчивость управляемых систем на ориентируемых поверхностях", Матем. сб., 182:1 (1991), 3-35; англ. пер.: А. А. Davydov, "Structural stability of control systems on orientable surfaces", Math. USSR-Sb., 72:1 (1992), 1-28.

[40] А.Ф. Филиппов, "Устойчивость для дифференциальных уравнений с разрывными и многозначными правыми частями", Дифферени. уравнения, 15:6 (1979), 1018-1027; англ. пер.: A.F. Filippov, "Stability for differential equations with discontinuous and multivalued right-hand sides", Differ. Equ., 15:6 (1979), 720-727.

А. А. Давыдов (А. А. Davydov)

Владимирский государственный университет им. А. Г. и Н. Г. Столетовых

E-mail: davydov@vlsu.ru

\section{В. М. Закалюкин (V. M. Zakalyukin)}

Московский государственный университет им. М. В. Ломоносова
Поступила в редакцию

14.10.2011 